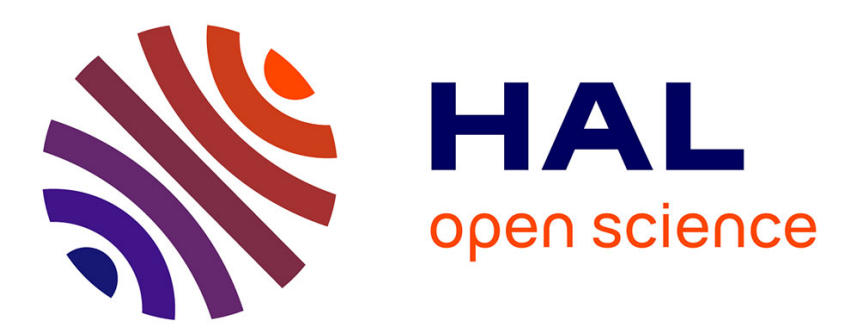

\title{
Blood Flow Simulations for the Design of Stented Valve Reducer in Enlarged Ventricular Outflow Tracts
}

\author{
Alfonso Caiazzo, Romain Guibert, Younes Boudjemline, Irene
}

Vignon-Clementel

\section{- To cite this version:}

Alfonso Caiazzo, Romain Guibert, Younes Boudjemline, Irene Vignon-Clementel. Blood Flow Simulations for the Design of Stented Valve Reducer in Enlarged Ventricular Outflow Tracts. Cardiovascular Engineering and Technology, 2015, 6 (4), pp.485-500. 10.1007/s13239-015-0240-z . hal-01244447

HAL Id: hal-01244447

https://hal.inria.fr/hal-01244447

Submitted on 15 Dec 2015

HAL is a multi-disciplinary open access archive for the deposit and dissemination of scientific research documents, whether they are published or not. The documents may come from teaching and research institutions in France or abroad, or from public or private research centers.
L'archive ouverte pluridisciplinaire HAL, est destinée au dépôt et à la diffusion de documents scientifiques de niveau recherche, publiés ou non, émanant des établissements d'enseignement et de recherche français ou étrangers, des laboratoires publics ou privés. 


\title{
Blood flow simulations for the design of stented valve reducer in enlarged ventricular outflow tracts
}

\author{
Alfonso Caiazzo $^{\mathrm{a}, *}$, Romain Guibert ${ }^{\mathrm{b}}$, Younes Boudjemline ${ }^{\mathrm{c}}$, Irene E. Vignon-Clementel ${ }^{\mathrm{d}}$ \\ ${ }^{a}$ Weierstrass Institute for Applied Analysis and Stochastics, Berlin, Germany \\ ${ }^{b}$ Université de Toulouse; INPT, UPS; Institut de Mécanique des Fluides de Toulouse, France \\ ${ }^{c}$ Service de Cardiologie Pédiatrique, Hôpital Necker-Enfants Malades, Paris, France \\ ${ }^{d}$ INRIA Paris-Rocquencourt and Sorbonne Universités UPMC Univ. Paris 6, Laboratoire J.-L. Lions, Paris, France
}

\begin{abstract}
Tetralogy of Fallot is a congenital heart disease characterized over time, after the initial repair, by the absence of a functioning pulmonary valve, which causes regurgitation, and by progressive enlargement of the right ventricle outflow tract (RVOT). Due to this pathological anatomy, available transcatheter valves are usually too small to be deployed there. To avoid surgical valve replacement, an alternative consists in implanting a reducer prior to or in combination with the valve. It has been shown in animal experiments to be promising, but with some limitations. The effect of a percutaneous pulmonary valve reducer on hemodynamics in enlarged RVOT is thus studied by computational modeling. To this aim, blood flow in the RVOT is modeled with CFD coupled to a simplified valve model and 0D downstream models. Simulations are performed in an image-based geometry and boundary conditions tuned to reproduce the pathological flow without the device. Different device designs are built and compared with the initial device-free state, or with the reducer alone. Results suggest that pressure loss is higher for the reducer alone than for the full device, and that the latter successfully restores hemodynamics to a healthy state and induces a more symmetric flow in the pulmonary arteries. Moreover, pressure forces on the reducer and on the valve have the same magnitudes. Migration would occur towards the right ventricle rather than the pulmonary arteries. Results support the thesis that the reducer does not introduce clinically significant pressure gradients, as was found in animal experiments. Such study could help transfer to patients.
\end{abstract}

Keywords: Device design, percutaneous pulmonary valve replacement, multi scale blood flow simulations, right ventricle outflow tract (RVOT), repaired Tetralogy of Fallot

${ }^{*}$ Correspondence to: Alfonso Caiazzo, Weierstrass Institute for Applied Analysis and Stochastics, Mohrenstrasse 39, 10117 Berlin, Germany

Email addresses: caiazzo@wias-berlin.de (Alfonso Caiazzo), romain.guibert@imft.fr (Romain Guibert), younes.boudjemline@nck.ap-hop-paris.fr (Younes Boudjemline), irene.vignon-clementel@inria.fr (Irene E. Vignon-Clementel) 


\section{Introduction}

Tetralogy of Fallot (ToF) is a severe congenital heart disease (CHD) characterized after the initial repair, by the absence of a functioning pulmonary valve. This causes blood flow regurgitation in the right ventricle during diastole, leading to progressive enlargement of the right ventricle, and pulmonary arteries. Valve replacement is thus often warranted at some course of the disease progression. Percutaneous pulmonary valve implantation (PPVI) has been developed to correct pulmonary insufficiency and stenosis, based on the implantation by catheterization of a biological valve sewn into a balloon expandable stent [6]. However valved stents (Melody valve, Medtronic Inc) are available in diameter up to $22 \mathrm{~mm}$ making PPVI impossible in native or patched enlarged right ventricle outflow tracts (RVOT) as seen in post-operative Fallot patients [8, 22, 25]. Yet, this population actually constitutes the vast majority of patients in need of valve replacement (75\%) [1]. Note that even if biological or manufactured valves would be available at these enlarged sizes (up to $50 \mathrm{~mm}$ ), percutaneous delivery and proper functionality in such complex anatomy would be very challenging. To avoid redo-surgery in these patients, some authors have advocated to create a landing zone by reducing the RVOT externally similar to a pulmonary artery (PA) banding or internally by implanting a stent that reduces the diameter of the PA $[1,8,24]$. These intravascular devices can be implanted together or in a two-step procedure where the reducer is first introduced alone to check that it does not introduce a clinically significant pulmonary pressure gradient. This self-expandable device has shown to be promising on animals, however several limitations were experienced. One of them comes from the fact that RVOT anatomies in patients that suffer from severe pulmonary regurgitation differ in diameter and length between the right ventricular outflow and the pulmonary artery bifurcation [29], and thus the original length of $55 \mathrm{~mm}$ [8] could only fit into a minority of patients. Shorter reducers (or at least in their cramped version) were designed [1,24], with different surface anchorages. Another issue is the complex patient anatomy that could effectively reduce the device diameter and create additional flow obstruction or valve malfunction. There is thus a need to better understand the relationship between device designs and hemodynamics in these anatomies. A further motivation to study hemodynamics is that they have been postulated being as important in provoking remodeling in the pulmonary vasculature as endothelial injury due to the device implantation [7].

To the authors' knowledge, very little biomechanics research has been carried out on PPIV, with or without reducer. Related works include the power loss calculation from patients catheterization and MRI data in RVOT after ToF repair, to propose a threshold for when to perform pulmonary valve replacement [14]. Stent fracture in PPVI, without reducer, has been investigated based on patient-specific solid mechanics simulations [30]. Connected computational blood flow simulations describe the fluid mechanics due to replacement of other types of valves (typically mechanical aortic valves, e.g. [39]) or on the hemodynamic forces that can explain the direction of stented graft migration in other arteries (e.g. the aorta [27]). Previous hemodynamics modeling works after ToF repair studied pulmonary regurgitation with OD [21] or 3D simulations [12, 19], but none of these have been performed yet in the context of this percutaneous pulmonary valve reducer (PPVR).

Since this device has not been implanted yet in patients, this work aims at providing a tool to predict hemodynamics changes due to PPVR for the enlarged right ventricular outflow tracts. It is 
in the spirit of earlier pulmonary flow investigations of surgical repair in CHD [36, 38]. The same approach could be applied for other heart valve functional replacements assessment [28]. In our previous work [19] hemodynamics in patient-specific repaired ToF pulmonary arteries, including some with enlarged outflow tracts, were simulated but without any device. In this study, the impact on hemodynamics of such a PPVR is for the first time investigated. Designs with varying diameters and lengths are considered. The impact of the reducer by itself is also investigated. Flow and pressure information are reported in the different configurations. The pressure gradient due to the device should indeed remain low in the clinical sense, in order to not create additional obstruction. Although it is expected to be low in these abnormally enlarged RVOTs, it could become more significant for the narrower and longer reducer designs. In addition, the pressure forces on the valve and on the reducer are quantified, as these represent a key factor for the device stability. The flow field is also described, as flow detachment fosters clot formation.

The rest of the paper is organized as follows. In Section 2 the computational methods of this study are described, including the generation of computational domains and the numerical methods for solving the Navier-Stokes equations, in the different device configurations. The next section presents simulation verifications, the hemodynamics results in the enlarged RVOT, without device, with the reducer alone and with the PPVR and the outcome of the device design study. Finally, results and limitations are discussed in Section 4, while the last section draws the conclusions on the device design and the proposed simulation approach.

\section{Materials and Methods}

This section describes the geometrical models, their meshing, and the computational methods simulate hemodynamics without or with valve.

\subsection{Computational geometries}

\subsubsection{Pulmonary artery segmentation and mesh generation}

A ToF patient surface representation of the pulmonary outflow tract and first pulmonary artery bifurcation is built from magnetic resonance images [23]. GHS3D ${ }^{1}$ [17] and TetGen [20] are used to generate the volume tetrahedral meshes. The resulting geometry consists of a segment of main pulmonary artery (MPA) with an inlet diameter of $27.2 \mathrm{~mm}$, and two branches of length $26 \mathrm{~mm}$ (left PA) and $34 \mathrm{~mm}$ (right PA), respectively, with outlet boundaries diameters of $19 \mathrm{~mm}$ (left PA) and $16 \mathrm{~mm}$ (right PA) - see Figure 1, left.

\subsubsection{Reducer and artificial valve modeling}

The reducer stent described in [24] (Figure 1, center) has a toroidal shape with an external diameter, before implantation, of $35 \mathrm{~mm}$, and a $15 \mathrm{~mm}$ internal diameter. A geometrical model of the reducer is created in 3-Matic ${ }^{2}$ as a torus geometry with given inner and outer radii, intersecting the original surface (Figure 1, right). For this study, the pulmonary valve is modeled as a resistive surface (with variable resistance) conformal to the mesh (as in [4], see Section 2.2.1 for more details on the numerical method), and drawn at the middle plane of the reducer geometry.

\footnotetext{
${ }^{1}$ https://www-roc.inria.fr/gamma/gamma/ghs3d/ghs.php

${ }^{2}$ http://biomedical.materialise.com/3-matic-0/
} 

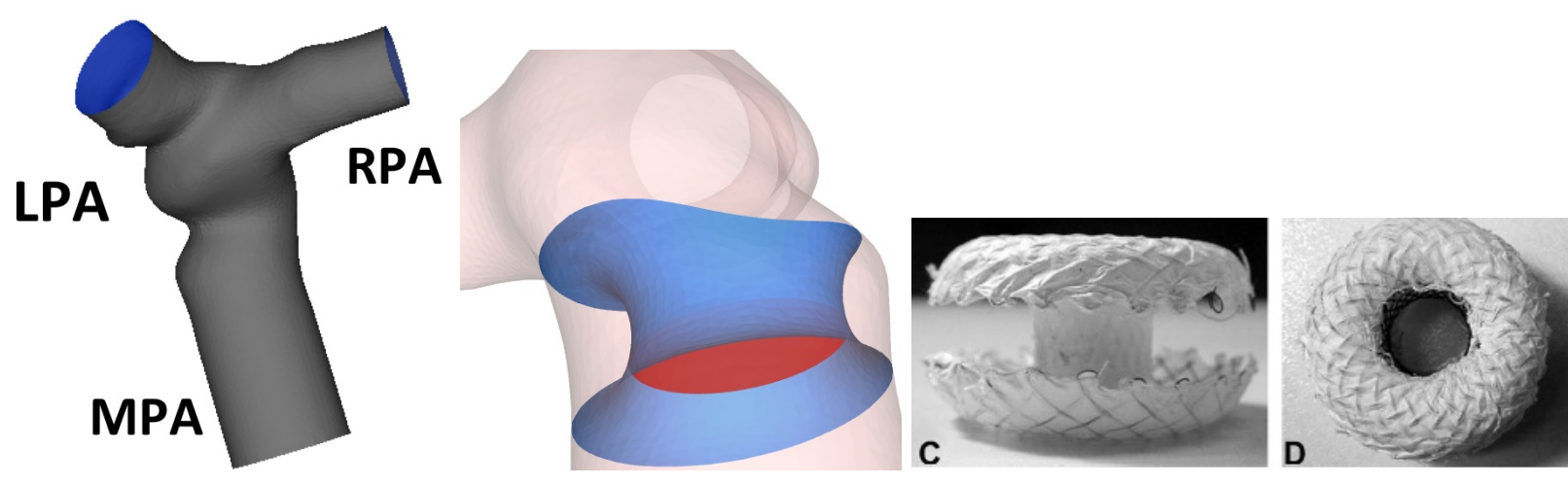

Figure 1: Left: the computational ToF patient surface, main pulmonary artery (MPA) and left (LPA) and right (RPA) pulmonary arteries. Center: computational model of the reducer (blue) as a torus intersecting the arterial wall (light pink), with given inner diameter and length, and closed valve (red). Right: two views of the coated reducer stent (pictures from [24]). The diameters (before implantation) are approximately $15 \mathrm{~mm}$ (inner) and $35 \mathrm{~mm}$ (outer), while the length is between 20 and $55 \mathrm{~mm}$.

\subsubsection{Variations of reducer length and diameter}

The original mesh is modified in order to generate different geometrical configurations (length and diameter) for the reducer. Length is varied, extending the reducer along its axis (in 3-Matic) and remeshing the obtained surfaces (Figure 2, top). Four different lengths are considered, from $22 \mathrm{~mm}$ to $37 \mathrm{~mm}$. Meshes with reducers of different diameters (from $14 \mathrm{~mm}$ up to $25 \mathrm{~mm}$ ) are obtained by smoothly deforming the configuration with device of length $27 \mathrm{~mm}$ and diameter $19 \mathrm{~mm}$, see Figure 2 (bottom). This is done by solving numerically a hyperelasticity problem on the original three-dimensional geometry (imagined as filled by an elastic material) and applying a surface normal force on the reducer surface. This approach allows to easily obtain a computational domain with a different reducer diameter as a one-to-one deformation of the original configuration, hence without the need of regenerating the three-dimensional mesh (see, e.g., [19]).

\subsection{Blood flow modeling}

The pulmonary arteries (PA) are described by a three-dimensional domain $\widehat{\Omega}$, which boundary is decomposed as

$$
\partial \widehat{\Omega}=\Sigma_{\text {in }} \cup \Sigma_{\text {wall }} \cup \Sigma_{\text {out }}
$$

denoting the inlet, the wall and the outlet boundaries, respectively.

Blood flow is modeled as an incompressible, Newtonian fluid, which is described by the incompressible Navier-Stokes equations in terms of velocity $\hat{\mathbf{u}}: \widehat{\Omega} \times \mathbb{R}^{+} \rightarrow \mathbb{R}^{d}$ and pressure $\hat{p}: \widehat{\Omega} \times \mathbb{R}^{+} \rightarrow \mathbb{R}$, leading with the boundary conditions to the system:

$$
\left\{\begin{aligned}
\rho \partial_{t} \hat{\mathbf{u}}+\rho \hat{\mathbf{u}} \cdot \nabla \hat{\mathbf{u}}+\nabla \hat{p}-2 \mu \operatorname{div} \boldsymbol{\epsilon}(\hat{\mathbf{u}})=0 & \text { in } \quad \widehat{\Omega}, \\
\operatorname{div} \hat{\mathbf{u}}=0 & \text { in } \quad \widehat{\Omega}, \\
\sigma(\hat{\mathbf{u}}, \hat{p}) \mathbf{n}=-p_{\text {in }} \mathbf{n} & \text { on } \quad \Sigma_{\text {in }} \\
\hat{\mathbf{u}}=0 & \text { on } \quad \Sigma_{\text {wall }}, \\
\sigma(\hat{\mathbf{u}}, \hat{p}) \mathbf{n}=-p_{\text {out }} \mathbf{n} & \text { on } \quad \Sigma_{\text {out }} . \\
4 &
\end{aligned}\right.
$$




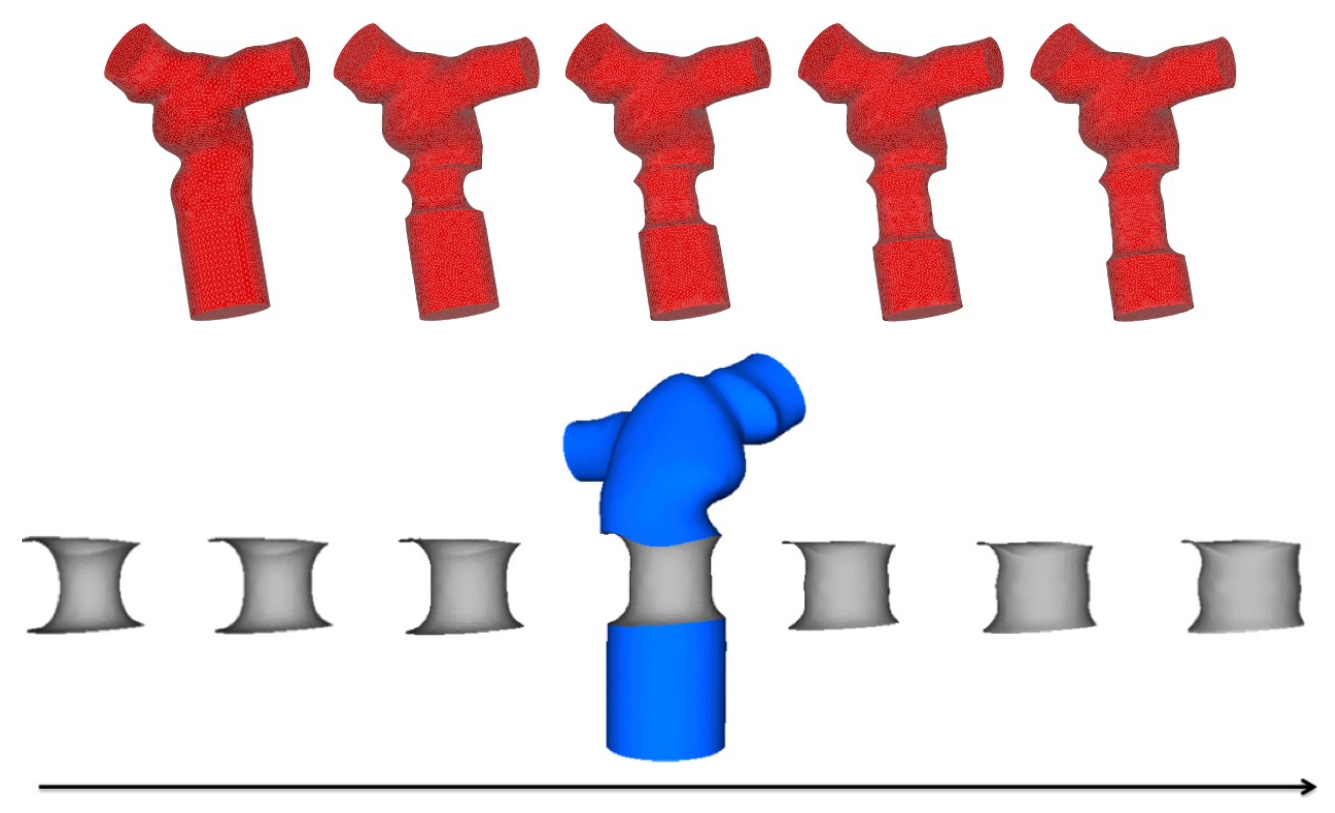

Figure 2: Top: computational 3D model without PPVR (leftmost picture) and with PPVR of length $22 \mathrm{~mm}$ to $37 \mathrm{~mm}$, diameter $19 \mathrm{~mm}$. Bottom: model of reducers of different diameters (14 $\mathrm{mm}$ to $25 \mathrm{~mm}$ ) obtained by deforming the geometry with reducer length $27 \mathrm{~mm}$ and diameter $19 \mathrm{~mm}$ (middle figure).

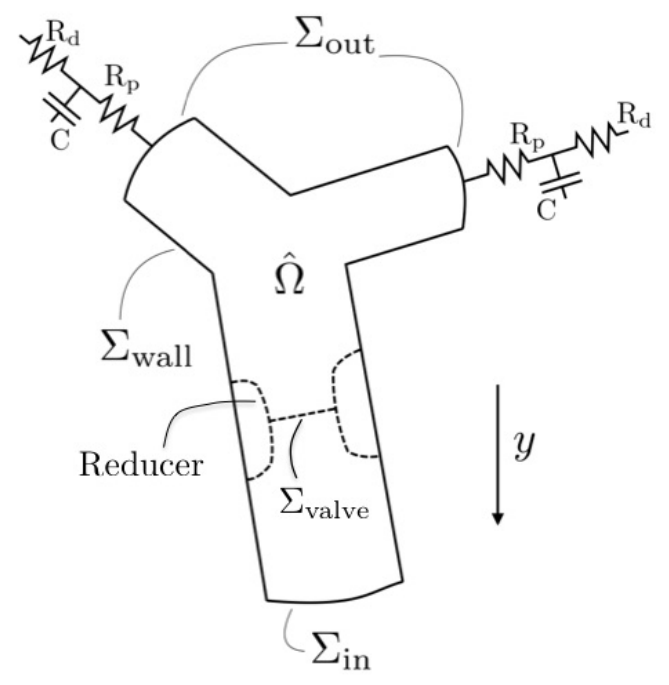

Figure 3: PA sketch including the inlet surface $\Sigma_{\text {in }}$, the wall $\Sigma_{\text {wall }}$, the reducer and its valve (represented in closed configuration) in dashed line, and the Windkessel boundary conditions (see Section 2.2.2) applied at the two outlets $\Sigma_{\text {out }}$. The $y$-axis is directed towards the right ventricle. 
In (2), $\rho$ stands for the density of the blood and $\sigma(\hat{\mathbf{u}}, \hat{p})$ denotes the fluid Cauchy-stress tensor defined by

$$
\boldsymbol{\sigma}(\mathbf{u}, p)=-p \mathbf{I}+2 \mu \epsilon(\mathbf{u}), \quad \boldsymbol{\epsilon}(\mathbf{u}) \stackrel{\text { def }}{=} \frac{1}{2}\left(\nabla \mathbf{u}+\nabla \mathbf{u}^{\mathrm{T}}\right),
$$

$\mu$ being the blood dynamic viscosity. In the numerical simulation, blood density and viscosity have been set to $\rho=1 \mathrm{~g} / \mathrm{cm}^{3}$ and $\mu=0.04$ Poise, respectively, yielding, for the considered flow regime, a Reynolds number $\operatorname{Re}=\frac{\rho U_{\operatorname{mean}} D_{\mathrm{PA}}}{\mu} \approx 2400$ and Womersley number Wo $=\frac{\omega D_{\mathrm{PA}}^{2}}{\mu} \approx 185$.

The boundary conditions are defined by inlet and outlet pressures $\left(p_{\text {in }}\right.$ and $p_{\text {out }}$ on $\Sigma_{\text {in }}$ and $\Sigma_{\text {out }}$, respectively), while no slip boundary conditions are imposed for the velocity on $\Sigma_{\text {wall }}$. Note that since the different reducers have a covered stent $[1,8,24]$, the reducer, when present, has been considered as part of the wall $\Sigma_{\text {wall }}$.

\subsubsection{Valve modeling}

The pulmonary valve is modeled as a resistive immersed surface (RIS) [4], i.e. considering the valve as a thin porous interface which resistance to flow depends on the current flow and pressure conditions across the valve surface, varying between zero (when the valve is open) to a very high value (when the valve is closed).

At the continuous level, the immersed surface results in an additional singular term in the Navier-Stokes equation, replacing equation $(2)_{1}$ with

$$
\rho \partial_{t} \hat{\mathbf{u}}+\rho \hat{\mathbf{u}} \cdot \nabla \hat{\mathbf{u}}+\nabla \hat{p}-2 \mu \operatorname{div} \boldsymbol{\epsilon}(\hat{\mathbf{u}})+r_{\mathrm{RIS}}(\hat{\mathbf{u}}, p) \hat{\mathbf{u}} \delta_{\Sigma_{\text {valve }}}=0 \quad \text { in } \quad \widehat{\Omega},
$$

denoting $\delta_{\Sigma_{\text {valve }}}$ the Dirac-delta function of the valve surface and with $r_{\mathrm{RIS}}(\hat{\mathbf{u}}, p)$ the surface resistance as a function of current flow conditions, varying between $r_{\mathrm{RIS}}(\hat{\mathbf{u}}, p)=0$ (valve is open) and $r_{\mathrm{RIS}}(\hat{\mathbf{u}}, p) \gg 1$ (valve closed).

The major advantage of this reduced approach compared to a fully-resolved valve model is that it drastically reduces the computational effort, as it requires neither a spatial resolution of the three dimensional valve geometry, nor a coupled fluid-structure solver that handles contact, while being very robust. In fact, in the finite element framework the valve is represented by a static two-dimensional surface conformal to the three dimensional finite element mesh.

Notice that, if $r_{\mathrm{RIS}}(\hat{\mathbf{u}}, p)>0$, the pressure across the valve interface is discontinuous, and equation (3) can be equivalently seen as two Navier-Stokes problems coupled by a proper pressure jump condition $[10,13]$.

Denoting with $\Delta p_{\text {valve }}$ this pressure jump, i.e. the pressure difference between the two faces of the simplified valve, and with $Q_{\text {valve }}$ the flow rate across the valve surface, the resistance function has been defined as follows:

$$
r_{\mathrm{RIS}}(\hat{\mathbf{u}}, p)= \begin{cases}10^{8} & \text { if valve is open and } Q_{\mathrm{valve}}<0 \\ 0 & \text { if valve is close and } \Delta p_{\text {valve }}>0 .\end{cases}
$$

i.e., the valve closes as soon as the flow becomes negative (directed towards the right ventricle) and reopens when the ventricular pressure is higher than the pressure in the PA.

As it was shown in [4], the model is able to reproduce a physiological pressure drop across the valve and to create very realistic jet and flow structures downstream of the valve. It was furthermore successfully tested in normal and disease conditions. 


\subsubsection{Inlet and outlet boundary conditions}

At the inlet boundary, a time-varying right ventricular pressure is prescribed uniformly on the inlet section. Although some measured inlet flow rates were available for the device-free condition, imposing the inlet pressure allows to maintain the same boundary condition for the device-free model and the other configurations, with the reducer alone and with the PPVI. In particular, this would not be possible with an inlet flow rate boundary condition, as this quantity drastically changes once the pulmonary valve is implanted. However, available flow measurements have been used for preliminary validation (see Section 3.1 and Discussion). At the outlets, a relationship between pressure and flow is prescribed, in order to take into account the effect of the pulmonary vasculature downstream of the $3 \mathrm{D}$ computational model. Notice that this boundary condition fixes neither the flow rate nor the pressure, which are both part of the desired solution and may thus change with the different designs.

Considering the outlet boundary composed of two disjointed surfaces, i.e. $\Sigma_{\text {out }}=\Gamma_{1} \cup \Gamma_{2}$, the outlet pressure $p_{\text {out }}^{i}$ at each outlet boundary $\Gamma_{i}$ is defined by a lumped parameter model, in which $p_{\text {out }}^{i}(t)$ is related to the outgoing flux at $\Gamma_{i}$ by a 3-element Windkessel model originally proposed in [15] (see, e.g., [35] for more recent applications in CHD pulmonary artery modeling):

$$
p_{\text {out }, i}+R_{\mathrm{d}} C \frac{\mathrm{d} p_{\text {out }, i}}{\mathrm{~d} t}=\left(R_{\mathrm{p}}+R_{\mathrm{d}}\right) Q_{\text {out }, i}+R_{\mathrm{p}} R_{\mathrm{d}} C \frac{\mathrm{d} Q_{\mathrm{out}, i}}{\mathrm{~d} t},
$$

(on each outlet boundary $\Gamma_{i}, i=1,2$ ), where

$$
Q_{\mathrm{out}, i}(t)=\int_{\Gamma_{i}} \mathbf{u}(t) \cdot \mathbf{n} d s
$$

$R_{\mathrm{p}}$ and $R_{\mathrm{d}}$ model the resistance of the proximal and distal vasculature, respectively, and the capacitance $C$ takes into account the ability of the downstream vessels to store blood during peak flow and recoil when pressure decreases. These parameters need then to be calibrated depending on available measurements [2, 26, 34] (see Section 3.1).

\subsubsection{Numerical approximation}

Problem (2) is solved numerically with a time-discretization based on a Chorin-Temam projection scheme (see, e.g., $[11,18,33]$ ) in which velocity and pressure are computed separately in two substeps. Namely, denoting with $\tau$ the time-step size and setting $t_{n} \stackrel{\text { def }}{=} n \tau$ for $1 \leq n \leq N$, for a given initial condition $\mathbf{u}^{0}=\mathbf{u}_{0}$, the time iteration consists of solving the two problems:

1. Velocity step:

$$
\left\{\begin{aligned}
\rho \frac{\hat{\mathbf{u}}^{n+1}-\hat{\mathbf{u}}^{n}}{\tau}+\rho \hat{\mathbf{u}}^{n} \cdot \boldsymbol{\nabla} \hat{\mathbf{u}}^{n+1}-2 \mu \boldsymbol{\nabla} \cdot \boldsymbol{\epsilon}\left(\hat{\mathbf{u}}^{n+1}\right) & \\
+r_{\mathrm{RIS}}\left(\hat{\mathbf{u}}^{n}, p^{n}\right) \delta_{\Sigma_{\text {valve }}} \hat{\mathbf{u}}^{n+1}+\nabla \hat{p}^{n} & =\mathbf{0}, \text { in } \widehat{\Omega}, \\
2 \mu \epsilon\left(\hat{\mathbf{u}}^{n+1}\right) \mathbf{n} & =\mathbf{0} \text { on } \Sigma_{\text {in }} \cup \Sigma_{\text {out }}, \\
\hat{\mathbf{u}}^{n} & =\mathbf{0} \text { on } \Sigma_{\text {wall }} .
\end{aligned}\right.
$$


2. Pressure-Poisson projection step:

$$
\left\{\begin{aligned}
-\frac{\tau}{\rho} \Delta \hat{p}^{n+1} & =-\boldsymbol{\nabla} \cdot \hat{\mathbf{u}}^{n+1} \text { in } \widehat{\Omega} \\
\hat{p}^{n+1} & =p_{\text {in }}^{n+1} \text { on } \Sigma_{\text {in }} \\
\frac{\tau}{\rho} \frac{\partial \hat{p}^{n+1}}{\partial \mathbf{n}} & =0 \text { on } \Sigma_{\text {wall }}, \\
\hat{p}^{n+1} & =p_{\text {out }}^{n+1} \text { on } \Sigma_{\text {out }} \\
\frac{r_{\mathrm{RIS} \tau} \tau}{\rho} \partial_{\mathbf{n}} p^{n+1}+\llbracket p^{n+1} \rrbracket & =r_{\mathrm{RIS}} \hat{\mathbf{u}}^{n} \cdot \mathbf{n}, \text { on } \Sigma_{\text {valve }}
\end{aligned}\right.
$$

In order to allow for discontinuous pressures across the valve during closure, the pressure degrees of freedom on the valve surface are doubled, i.e. doubling the vertices of the mesh belonging to

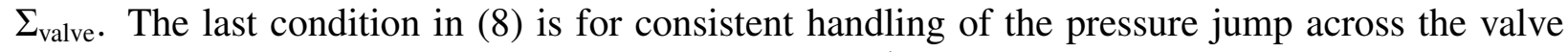
interface (see [10] for details). The outlet pressure $p_{\text {out }}^{n+1}$ is computed at each iteration discretizing (5) with an implicit Euler scheme and treating the 3D-0D coupling on the outlet boundary $i$ as follows:

$$
\begin{aligned}
&\left(1+\frac{R_{\mathrm{d}} C}{\tau}\right) p_{\mathrm{out}, i}^{n+1}-\frac{R_{\mathrm{d}} C}{\tau} p_{\mathrm{out}, i}^{n}= \\
&\left(R_{\mathrm{p}}+R_{\mathrm{d}}\right) Q_{\mathrm{out}, i}+R_{\mathrm{p}} R_{\mathrm{d}} C \frac{Q_{\mathrm{out}, i}^{n+1}-Q_{\mathrm{out}, i}^{n}}{\tau},
\end{aligned}
$$

where the outlet flux $Q_{\mathrm{out}, i}^{n+1}:=Q_{\mathrm{out}, i}\left(t^{n+1}\right)$ is computed from the latest solution of (7). The discretization in space of (7)-(8) is performed via continuous piecewise affine finite elements spaces $\left(\mathbb{P}_{1}\right.$ on tetrahedra) for both velocity and pressure, denoted by $\boldsymbol{V}_{h}$ and $Q_{h}$, respectively. In the resulting finite element formulation, the resistive term is treated numerically with the stabilized finite element method proposed and analyzed in [10]. Moreover, a tangential regularization [5] was used at the Neumann boundaries in equation (7) to prevent backflow instabilities.

\subsection{Simulations outputs}

The simulation results compare the different physiological states and device designs. Flow and pressure (averaged over boundaries) are plotted according to their variation in time, or given as average-in-time values. To ease comparison with literature, mean-over-time flows $\left(Q_{\text {mean }}\right)$ are reported in $\mathrm{L} / \mathrm{min}$, while extrema are given in $\mathrm{mL} / \mathrm{s}$. Pressure drops $\Delta P_{l}$ and $\Delta P_{r}$ are computed as the maximum difference between the inlet average pressure and the left or right average outlet pressure when the valve is open, while peak differences $\Delta_{\max } P_{l, r}=\max P_{\text {inlet }}-\max P_{l, r}$ are computed as the difference in peak pressures between inlet and outlets. More detailed quantities such as velocity magnitude and vectors, or vorticity magnitude are plotted on representative 3D-cuts. Pressure forces are computed on the wall, the reducer or the valve, as

$$
\mathbf{F}(t)=\int_{\Gamma} p(\mathbf{x}, t) \mathbf{n}(\mathbf{x}) d \Gamma
$$

where $\Gamma$ denotes the considered surface and $\mathbf{n}$ stands for the outgoing normal. Either its components or its magnitude are reported. For the magnitude, the maximum value in time and the mean over the cardiac cycle will be noted $F_{\max }$ and $F_{\text {mean }}$ respectively. 


\section{Results}

\subsection{Preliminary verification and validation}

\subsubsection{Results consistency without the device}

As flow and pressure measurements were not available for a specific patient, the simulation was set up to be representative of this long-term repaired ToF physiology. The Navier-Stokes equations were thus solved imposing as inlet boundary condition the right ventricular pressure obtained by catheterization from our medical expert for a typical patient (Figure 4, left). Three 2D Phase-Contrast Magnetic Resonance Imaging (MRI) main pulmonary arterial flows were available for such ToF patients. The parameters for the Windkessel models at the outlet boundaries were thus tuned in order to approximate these measurements at the inlet (Figure 4, center). As no branch flows were available, the left and right PA downstream trees were hypothesized to be symmetric. The following values were thus obtained for the two Windkessel models:

$$
R_{\mathrm{p}}=40 \mathrm{dyn} \cdot \mathrm{s} \cdot \mathrm{cm}^{-5} ; R_{\mathrm{d}}=420 \mathrm{dyn} \cdot \mathrm{s} \cdot \mathrm{cm}^{-5} ; C=0.004 \mathrm{~cm}^{5} \cdot \mathrm{dyn}^{-1} .
$$

The Navier-Stokes equations in the projection scheme formulation (7)-(8) were solved on a $158 \mathrm{~K}$ tetrahedra mesh (Figure 3, left), with a time step $\tau=0.001 \mathrm{~s}$.


Figure 4: Left: prescribed inlet pressure. Center: computed inlet flow rate (red, continuous line) vs. three different available patient data $(\times,+$ and $*$, in blue). Right: computed outlet flow rates.

Flow at the inlet exhibits the typical high forward flow followed by negative flow due to pulmonary inefficiency. Regurgitation fractions for the inlet, left and right PA are 67\%, 60\% and 57\%, respectively. The results are coherent with the available measurements. For further validation by comparison with the MRI literature, see the discussion section.

\subsubsection{Mesh independence study}

As a verification step, in order to quantify the influence of the discretization on the results, numerical simulations with three different levels of mesh refinement were run, comparing the outputs in terms of flow rates and mean pressures at the boundaries. Namely, three meshes were considered, with $158 \mathrm{~K}, 458 \mathrm{~K}$ and $670 \mathrm{~K}$ tetrahedras of characteristic size $1.5 \mathrm{~mm}, 0.9 \mathrm{~mm}$ and $0.7 \mathrm{~mm}$ respectively. The maximum difference during the cardiac cycle between the coarsest and the finest results is $3 \%$ for the inlet and outlet flow rates, and similarly for the outlet pressure profiles. In view of these results, only the coarsest mesh has been used next. 


\subsection{Effect of the device}

\subsubsection{Flow and pressure waveforms}

Next, we present the detailed numerical results for the hemodynamics without the device and with the PPVR which reducer is $22 \mathrm{~mm}$ in length and $19 \mathrm{~mm}$ in diameter (Figure 2, top row, first and second geometries from the left, respectively). In order to better understand the influences of the different device components (the reducer stent and the valve), we also performed numerical simulations considering the presence of a reducer, but without valve. In all cases the same right ventricular pressure was imposed as inlet boundary condition (Figure 4, left), choosing the Windkessel parameters at the outlet boundaries as detailed in Section 3.1.
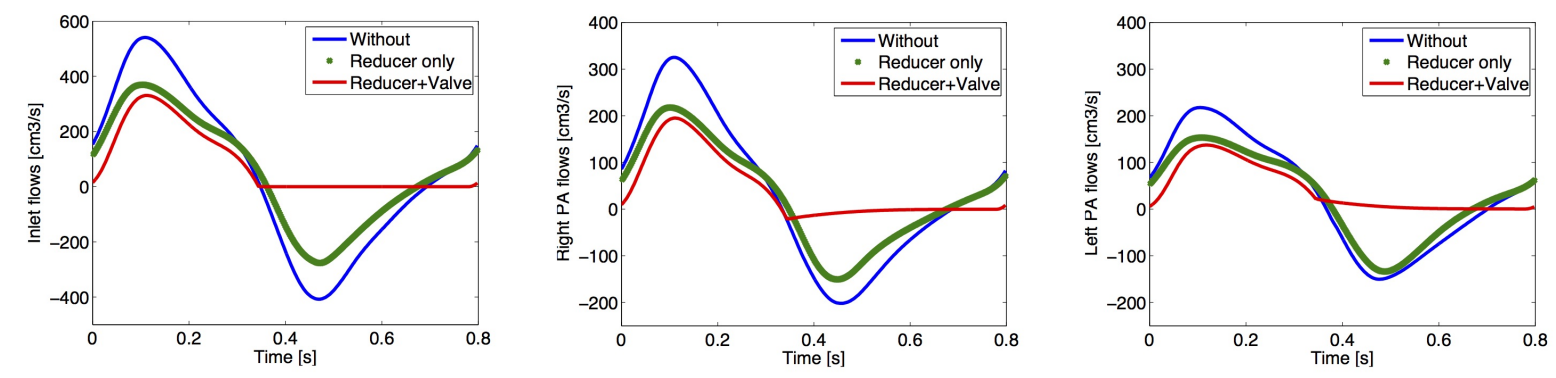

Figure 5: Comparisons of flow rates without device, with the reducer of length $22 \mathrm{~mm}$ and diameter $19 \mathrm{~mm}$, and with the PPVR at inlet (left), left PA (center), right PA (right).
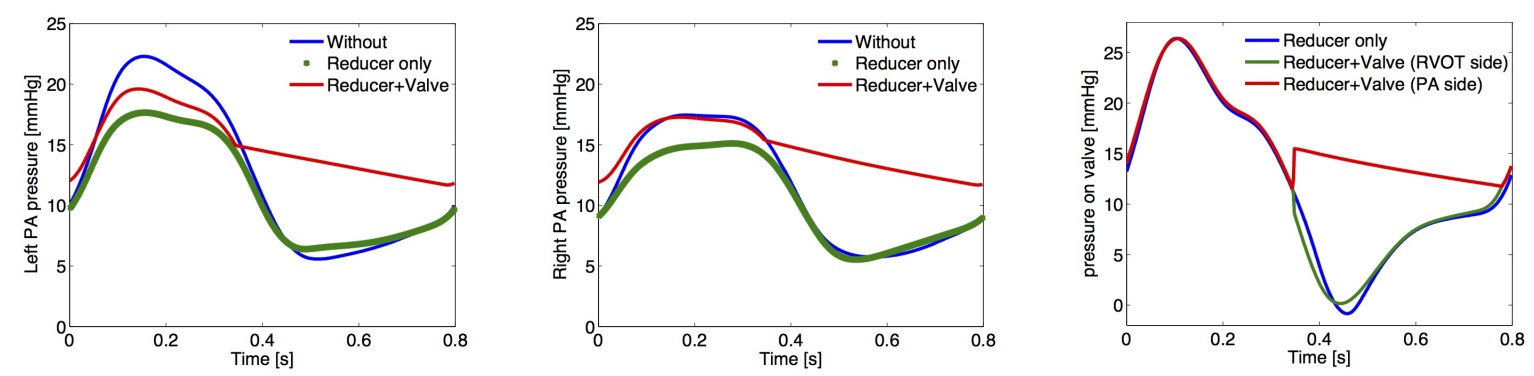

Figure 6: Comparisons of outlet pressures without device, with the reducer of length $22 \mathrm{~mm}$ and diameter $19 \mathrm{~mm}$, and with the PPVR at left PA (left), right PA (center) and on the valve (right). In the latter, the pressure for the reducer only case is computed in its middle plane, i.e. the location of the valve in the PPVR.

The computed inlet and outlet flow rates are shown for the three cases in Figure 5, while the outlet pressures are depicted in Figure 6. Without the device, the average inlet flow rate is $3.5 \mathrm{~L} / \mathrm{min}$, with a regurgitation of $67 \%$, while outlet flow rates are $1.9 \mathrm{~L} / \mathrm{min}(\mathrm{LPA})$ and $1.6 \mathrm{~L} / \mathrm{min}$ (RPA). The inlet flow rate peaks towards the PA at $500 \mathrm{~mL} / \mathrm{s}$ and flows back into the right ventricle (RV) with a maximum value of $370 \mathrm{~mL} / \mathrm{s}$. With the reducer alone, the average inlet flow remains $3.5 \mathrm{~L} / \mathrm{min}$, with a regurgitation of $65 \%$. However, the distribution among the two outlets becomes 
more symmetric ( $1.8 \mathrm{~L} / \mathrm{min}$ for the LPA and $1.7 \mathrm{~L} / \mathrm{min}$ for the RPA), corresponding also to more similar flow patterns in the two branches (Figure 7, center). With the full device, the average inlet flow rate increases to $5.0 \mathrm{~L} / \mathrm{min}$, equally distributed through each outlet boundary $(2.55 \mathrm{~L} / \mathrm{min}$ through the LPA and $2.45 \mathrm{~L} / \mathrm{min}$ through the RPA). There is no longer reverse flow at the inlet, while the left PA only has $6 \%$ of reverse flow. Maximum inlet flow is reduced to $330 \mathrm{~mL} / \mathrm{s}$. The different regurgitation amounts are summarized in Table 1.

Without any device, pressure in the PAs is lower than the inlet pressure during systole. Following first the decrease of the latter in diastole, it becomes higher than it, coherently with the significant amount of flow regurgitation. Pressure in the RPA is lower than in the LPA almost throughout the cardiac cycle, with a peak systolic difference close to $5 \mathrm{mmHg}$, denoting an asymmetric flow split between the two PAs. The differences between inlet and RPA and LPA maximum pressures are $12.5 \mathrm{mmHg}$ and $7.7 \mathrm{~mm} \mathrm{Hg}$, respectively.

With the reducer alone, diastolic pressures are almost the same as without the device, while systolic pressures are scaled down due to the additional resistance introduced by the reducer. Differences between inlet and RPA and LPA maximum pressures increase to $14.9 \mathrm{mmHg}$ and 12.4 $\mathrm{mmHg}$, respectively.

With the PPVR, these pressure waveforms are similar as before in systole, but then change shape: when the valve is closed, pressure is slowly relaxing down to $12 \mathrm{mmHg}$. They also become more similar between the two sides (peak difference below $3 \mathrm{mmHg}$ ), coherently with the more even symmetrical flow observed between the two PAs (Figure 7, right). The differences in maximum pressures between inlet and RPA and LPA are of 12.7 and 10.4, respectively. Inlet pressure leads inlet flow for all cases (Figures 4 and 5), whereas pressure is delayed in the PAs by 0.05 s (Figures 4 and 6). On the valve, pressure dynamics follows the inlet pressure on its upstream surface. On its part facing the PAs, it is the same when opened, but then almost exactly follows the two PA pressure waveforms during the rest of the cardiac cycle (Figure 6, right).

\begin{tabular}{|c|c|c|c|}
\hline & Without device & With reducer & With reducer + valve \\
\hline Inlet & $67 \%$ & $65 \%$ & $0 \%$ \\
\hline Left PA & $60 \%$ & $66 \%$ & $6 \%$ \\
\hline Right PA & $57 \%$ & $63 \%$ & $0 \%$ \\
\hline
\end{tabular}

Table 1: Regurgitation for the cases without device, with reducer only (length $22 \mathrm{~mm}$ and diameter $19 \mathrm{~mm}$ ) and with PPVR.

\subsubsection{Velocity, vorticity and pressure forces}

Figure 7 shows a few snapshots of the velocity magnitude in the three configurations, at different time instants (systole, diastole and intermediate time steps), while Figure 8 displays the magnitude of the vorticity, in different cuts selected to see the structures in the pulmonary artery trunk a.k.a main pulmonary artery (MPA), near the bifurcation and in the branches.

For all cases, the velocity field is streamlined forward into the PA trunk during most systole (Figures 7-8, first two rows), with low velocity regions around the PA bifurcation lower part (Figure 7 , first two rows). With the device, velocity remains high in the PA upper parts. Vorticity is 


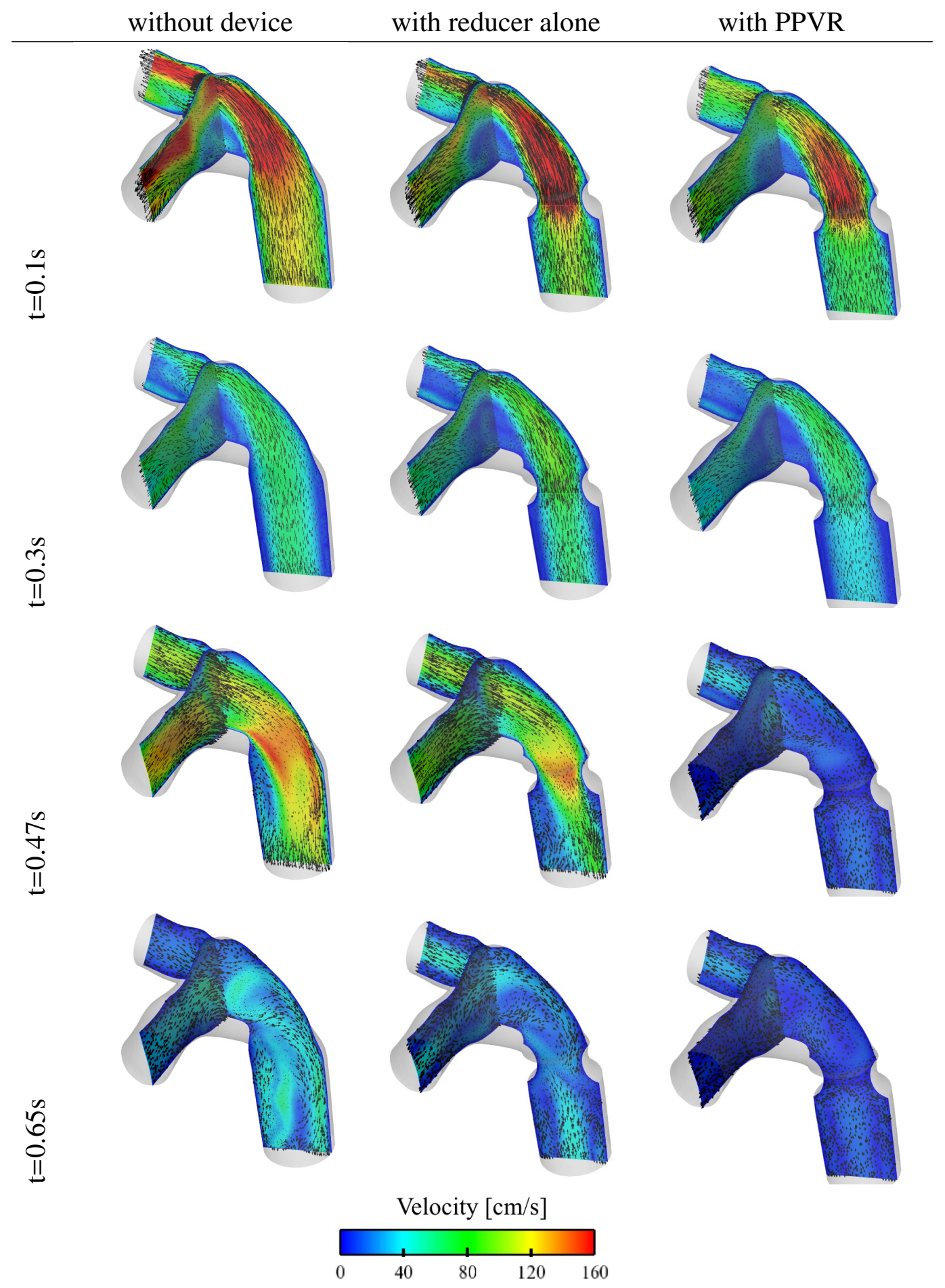

Figure 7: Velocity field snapshots on longitudinal cuts at selected instants (from top to bottom: $t=0.1,0.3,0.47,0.65$ s) during the cardiac cycle. Left: without device. Center: with reducer alone of length $22 \mathrm{~mm}$ and diameter $19 \mathrm{~mm}$. Right: with a PPVR of the same size. 
without device

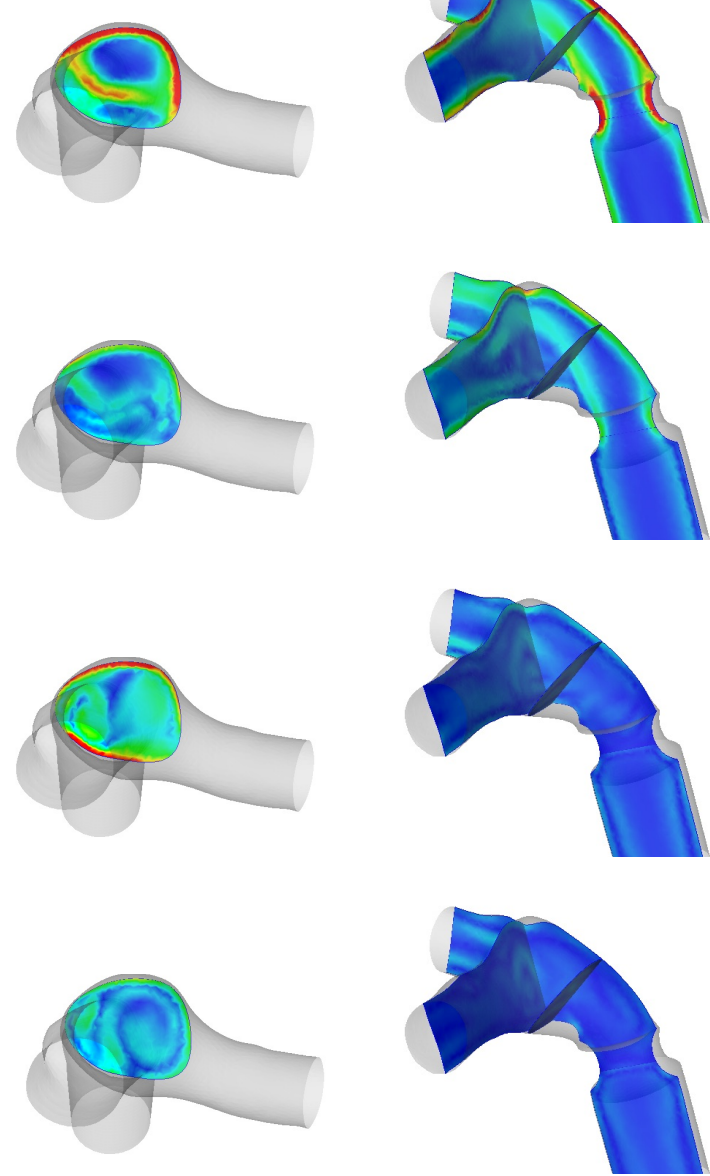

with PPVR
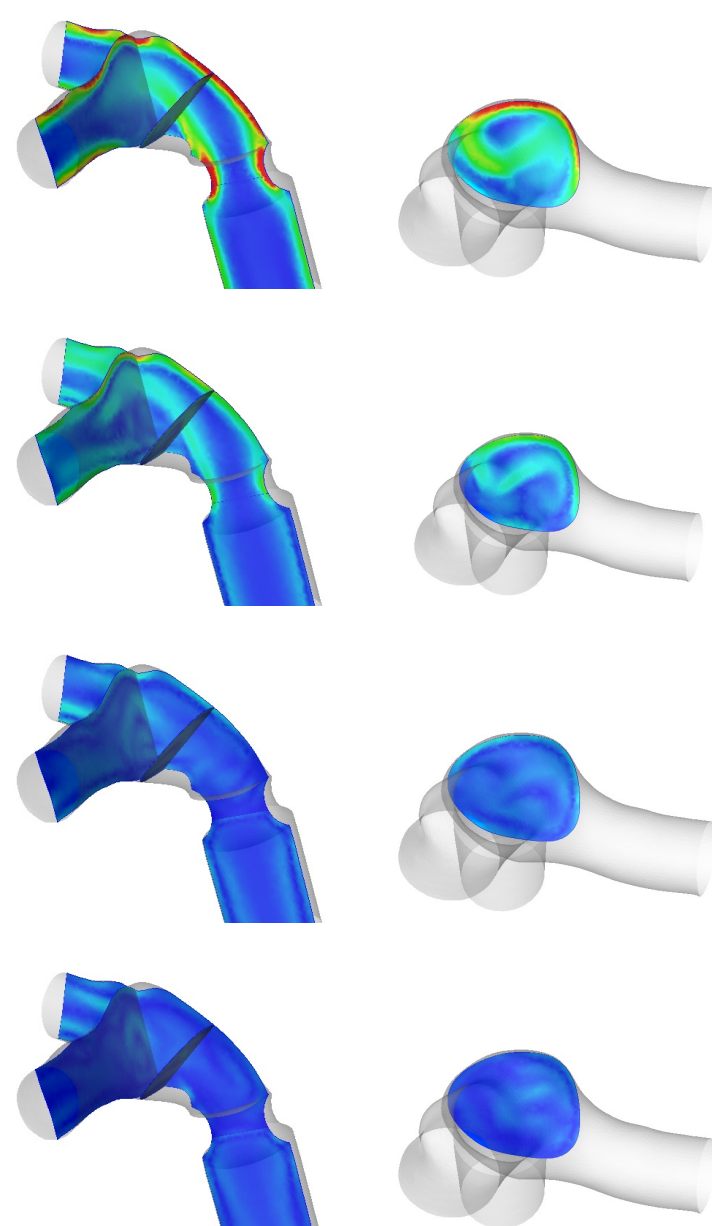

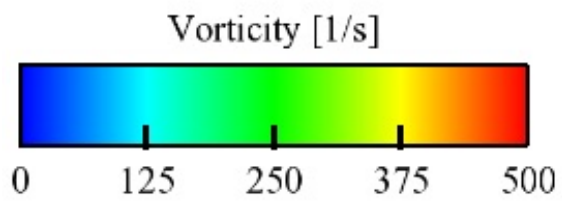

Figure 8: Vorticity magnitude snapshots on longitudinal and axial cuts at selected instants (from top to bottom: $t=$ $0.1,0.3,0.47,0.65 \mathrm{~s}$ ) during the cardiac cycle. Left: without device. Right: with a PPVR of length $22 \mathrm{~mm}$ and diameter $19 \mathrm{~mm}$. 
highest around the bifurcation and in the PAs. When flow reverses, the velocity field significantly changes: flow is parabolic in the PAs, but detaches in the posterior part of the MPA, and there is significant vorticity throughout the domain (Figure 7-8, left third rows). As the flow retracts from going down the RV, velocity is swirling in the bifurcation and in the MPA, with complex vorticity (Figure 7-8, left fourth rows). By contrast, with the PPVR, during forward flow, flow is accelerated as it goes through the device but it goes quite smoothly into the PAs (more homogeneous velocity field and lower vorticity in Figures $7-8$, right, first two rows). When the valve closes, the velocity field has little inertia (Figures 7-8, right last two rows). With the reducer only (Figure 7, center), the patterns during forward flow are similar to the full device case but with higher velocity magnitudes. During reverse flow, patterns are closer to the no device case, although they are more symmetric between left and right PAs, and the posterior flow detachment in the MPA is more pronounced.

Furthermore, the pressure forces on the device have been computed (see Figure 9), obtaining a maximum force (in magnitude) of $144 \mathrm{~N}$, and a mean force of $57 \mathrm{~N}$, with the maximum force corresponding to the minimum imposed RV pressure. The two lateral forces (in directions $x$ and $z$ ) are symmetric, indicating an axisymmetric radial force, pushing on the device during systole and unloading the device when the valve is closed, with the same order of magnitude. The axial force ( $y$-component) is dominating the other components, following mostly the dynamics of the imposed inlet pressure dynamics, but with different peak magnitudes, as the peak diastolic force is around 2.5 times the opposite peak systolic one. In fact, during systole, the force pushes on the reducer away from the RV, whereas when the valve is closed, this force pushes the device down towards the RV, equally on the reducer and on the valve.
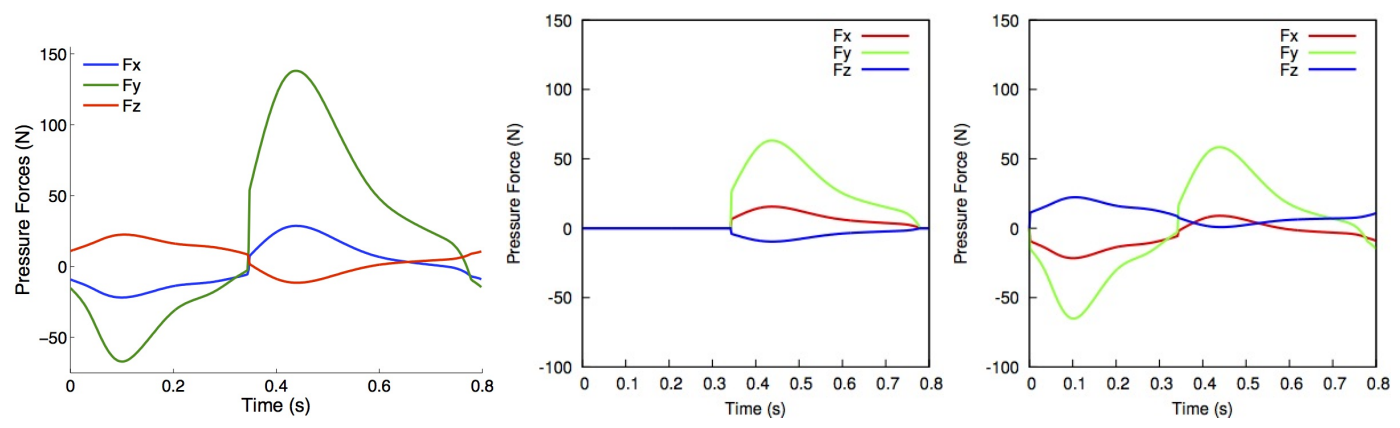

Figure 9: Resulting directional pressure forces over one cardiac cycle on the PPVR (reducer+valve) (left), on the valve alone (middle) and on the reducer (right). Note that the $y$-axis is directed from the PA towards the RV.

\subsection{Design study}

\subsubsection{Variation of flow direction}

Before studying the effect of geometrical parameter changes, a robustness test is performed. It assesses how sensitive the results are to the choice of inflow direction, as here the real velocity or pressure spatial inhomogeneity coming from the upstream right ventricle is not known. The effect on the hemodynamics of a change of flow direction at the inlet is thus studied, simulated 
by prescribing the inlet pressure on a tilted surface (at 45 degrees). Although the detailed velocity field changes, the pressure gradient remains almost unchanged and the radial force does not change significantly either (see Figure 10): the maximum force on the device is 147 N, i.e. an increase of only $2 \%$. Differences in maximum pressures between the inlet and the PAs are respectively $12.3 \mathrm{mmHg}$ (RPA) and $10 \mathrm{mmHg}$ (LPA). A slight increase in inlet flow rate is also observed (5.14 $\mathrm{L} / \mathrm{min},+2.8 \%$ ), probably due to complex three-dimensional effects.
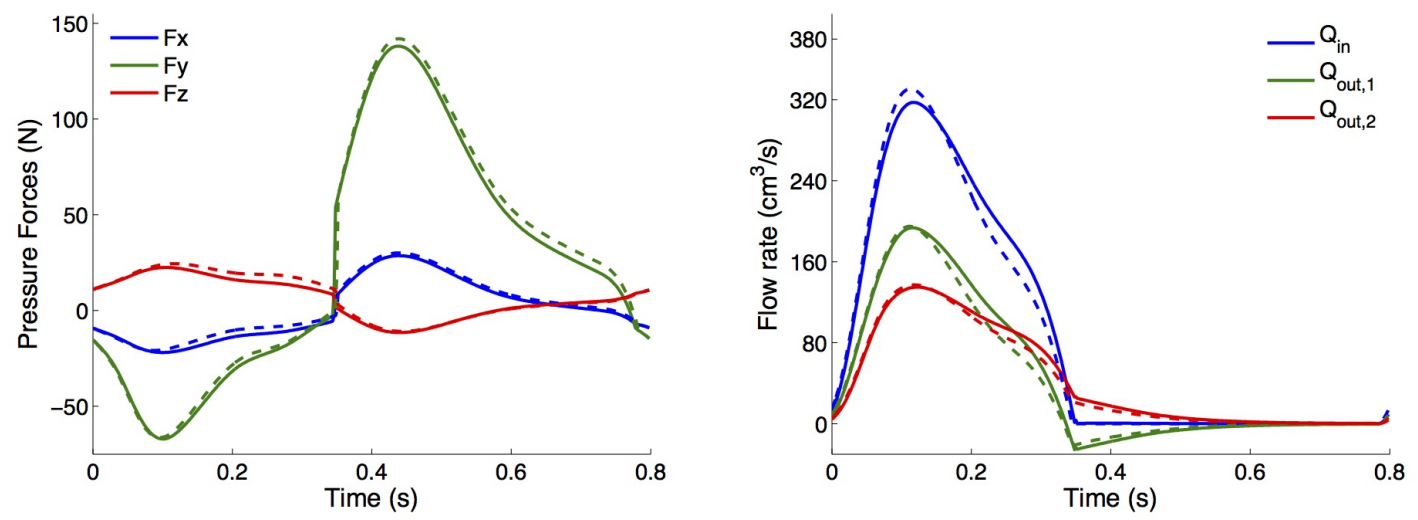

Figure 10: Left: Pressure forces for the configuration with the tilted inlet flow (dashed line) and for the reference device case (continuous line). Right: Flow rates for the configuration with the tilted inlet flow (dashed line) and for the reference device case (continuous line).

\subsubsection{Variation of reducer length}

In order to study the influence on hemodynamics of varying the device design, reducers of different lengths from $22 \mathrm{~mm}$ to $37 \mathrm{~mm}$ (see Figure 2, top row) were simulated. In all cases, the same time-varying inlet pressure and the same outlet Windkessel parameters were imposed as boundary conditions.

Figure 11 shows the forces on the device and the corresponding inlet and outlet flows, over one cardiac cycle. Table 2 summarizes the maximum and mean values of forces and mean flow rates for the different cases.

For all the different designs, the dynamics of forces and flow rates over the cardiac cycle are very similar. Lengthening the reducer decreases the maximum and mean forces. However, this correlation does not seem to be monotonically decreasing, as in the longest configuration forces are slightly larger. The mean inflow only experiences minor variations with respect to the original reducer length (increase between 2\% and 3\%). Finally, the reducer length does not affect the difference in maximum pressures across the PAs, which remain around $10-13 \mathrm{mmHg}$ (or $12-$ $14 \mathrm{mmHg}$ for the pressure drops).

\subsubsection{Variation of reducer diameter}

Next, the effect of the device diameter variations from $14 \mathrm{~mm}$ up to $25 \mathrm{~mm}$ (see Figure 2, bottom), was investigated, considering a reducer length of $27 \mathrm{~mm}$. In all cases, the same boundary conditions were applied as before. 

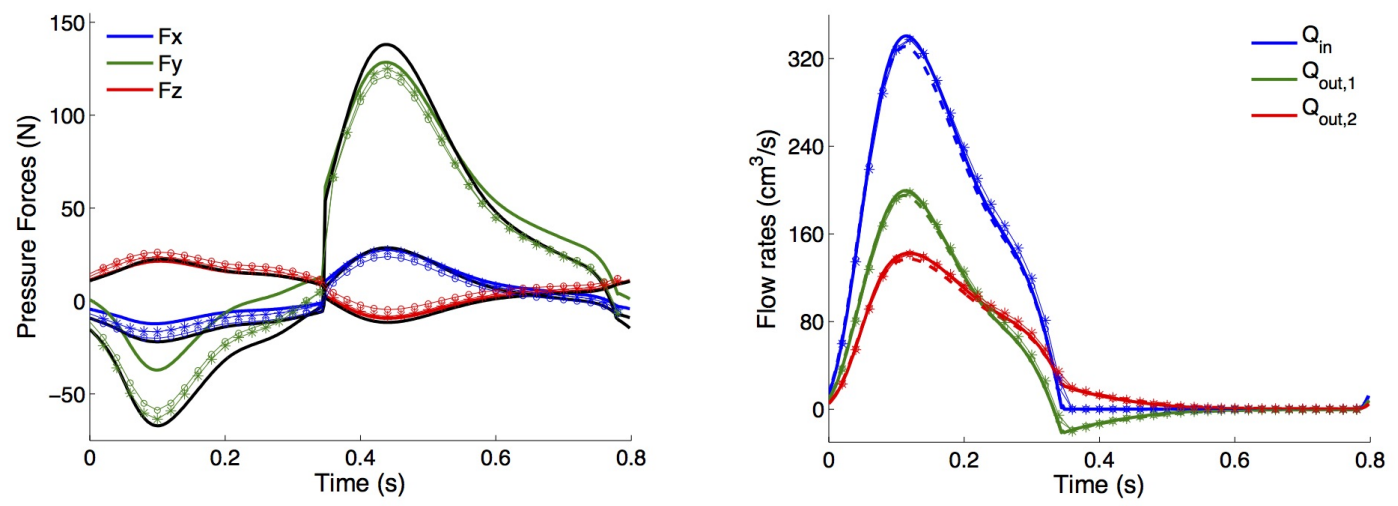

Figure 11: Resulting directional pressure forces acting on the device (left) and flow rates (right) over one cardiac cycle for different reducer lengths. Legend: black line: $22 \mathrm{~mm}$; continuous line: $27 \mathrm{~mm}$; circles: $32 \mathrm{~mm}$; stars: $37 \mathrm{~mm}$.
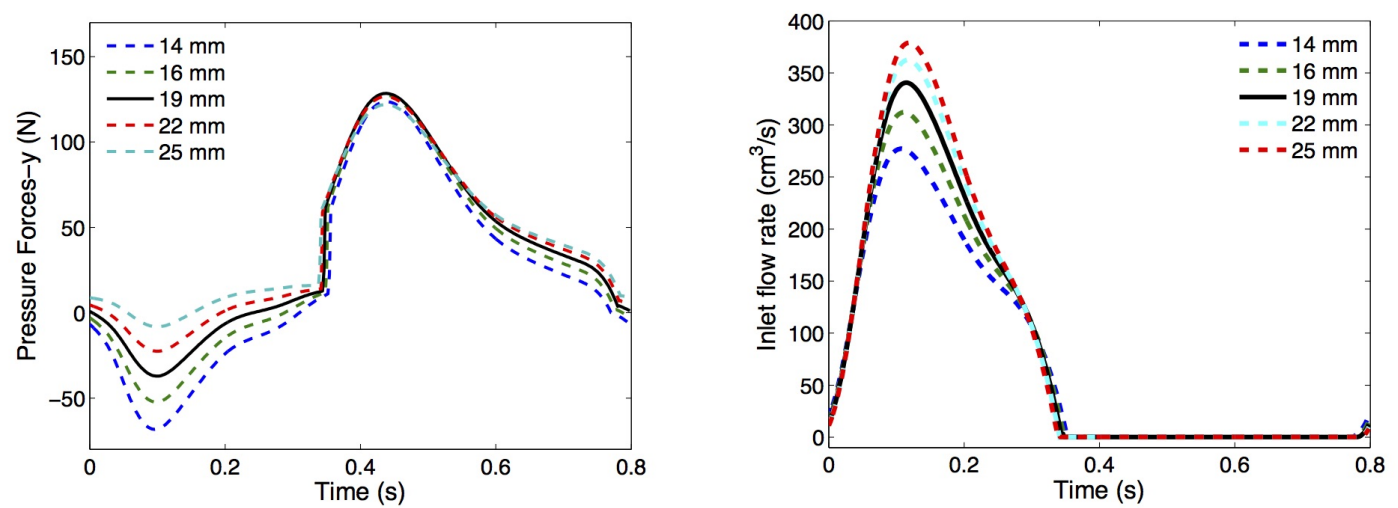

Figure 12: $y$-component of the pressure forces (along the PA axis) on the reducer (left) and flow rates (right), over one cardiac cycle for different stent diameters. Length is $27 \mathrm{~mm}$. 


\begin{tabular}{|c|c|c|c|c|}
\hline Length & $22 \mathrm{~mm}$ & $27 \mathrm{~mm}$ & $32 \mathrm{~mm}$ & $37 \mathrm{~mm}$ \\
\hline$F_{\max }(\mathrm{N})$ & 143.9 & $133.7(-7 \%)$ & $126.5(-12 \%)$ & $130.8(-9 \%)$ \\
\hline$F_{\text {mean }}(\mathrm{N})$ & 57.2 & $50.5(-12 \%)$ & $52.0(-9 \%)$ & $52.8(-8 \%)$ \\
\hline$Q_{\text {mean }}(\mathrm{L} / \mathrm{min})$ & 5.00 & $5.09(+2 \%)$ & $5.10(+2 \%)$ & $5.17(+3 \%)$ \\
\hline$\Delta P_{r}(\mathrm{mmHg})$ & 13.9 & $13.8(-0 \%)$ & $13.8(-0 \%)$ & $13.9(+0 \%)$ \\
\hline$\Delta_{\max } P_{r}(\mathrm{mmHg})$ & 12.7 & $12.4(-2 \%)$ & $12.5(-2 \%)$ & $12.4(-2 \%)$ \\
\hline$\Delta P_{l}(\mathrm{mmHg})$ & 12.0 & $12.1(+1 \%)$ & $12.1(+1 \%)$ & $12.2(+2 \%)$ \\
\hline$\Delta_{\max } P_{l}(\mathrm{mmHg})$ & 10.4 & $10.1(-3 \%)$ & $10.2(-2 \%)$ & $10.2(-2 \%)$ \\
\hline
\end{tabular}

Table 2: Maximum $\left(F_{\max }\right)$ and mean $\left(F_{\text {mean }}\right)$ forces on the device, mean inlet flow $\left(Q_{\text {mean }}\right)$, pressure drops across the two branches $\left(\Delta P_{l}\right.$ and $\left.\Delta P_{r}\right)$ and difference in maximum pressures $\left(\Delta_{\max } P_{l}\right.$ and $\left.\Delta_{\max } P_{r}\right)$ for different reducer lengths. Values are reported as variation with respect to the geometry with reducer length of $22 \mathrm{~mm}$. Diameter is $19 \mathrm{~mm}$.

\begin{tabular}{|c|c|c|c|c|c|c|}
\cline { 2 - 7 } \multicolumn{1}{c|}{} & $\oslash 14 \mathrm{~mm}$ & $\oslash 16 \mathrm{~mm}$ & $\oslash 17 \mathrm{~mm}$ & $\oslash 19 \mathrm{~mm}$ & $\oslash 22 \mathrm{~mm}$ & $\oslash 25 \mathrm{~mm}$ \\
\hline$F_{\max }(\mathrm{N})$ & $128.6(-4 \%)$ & $132.3(-1 \%)$ & $134.1(+0 \%)$ & 133.7 & $130.8(-2 \%)$ & $125.3(-6 \%)$ \\
\hline$F_{\text {mean }}(\mathrm{N})$ & $53.2(+5 \%)$ & $52.4(+4 \%)$ & $51.5(+2 \%)$ & 50.5 & $49.3(-2 \%)$ & $48.4(-4 \%)$ \\
\hline$Q_{\text {mean }}(\mathrm{L} / \mathrm{min})$ & $3.93(-23 \%)$ & $4.41(-13 \%)$ & $4.80(-6 \%)$ & 5.09 & $5.31(+4 \%)$ & $5.46(+7 \%)$ \\
\hline$\Delta P_{r}(\mathrm{mmHg})$ & $15.9(+15 \%)$ & $15.0(+9 \%)$ & $14.3(+4 \%)$ & 13.8 & $13.4(-3 \%)$ & $13.1(-5 \%)$ \\
\hline$\Delta_{\max } P_{r}(\mathrm{mmHg})$ & $15.3(+23 \%)$ & $14.2(+14 \%)$ & $13.2(+6 \%)$ & 12.4 & $11.8(-5 \%)$ & $11.4(-8 \%)$ \\
\hline$\Delta P_{l}(\mathrm{mmHg})$ & $14.5(+21 \%)$ & $13.4(+11 \%)$ & $12.6(+5 \%)$ & 12.1 & $11.6(-3 \%)$ & $11.3(-6 \%)$ \\
\hline$\Delta_{\max } P_{l}(\mathrm{mmHg})$ & $13.9(+37 \%)$ & $12.5(+23 \%)$ & $11.2(+10 \%)$ & 10.1 & $9.3(-8 \%)$ & $8.7(-14 \%)$ \\
\hline
\end{tabular}

Table 3: Maximum $\left(F_{\max }\right)$ and mean $\left(F_{\text {mean }}\right)$ forces on the device, mean inlet flow $\left(Q_{\text {mean }}\right)$, pressure drops across the two branches $\left(\Delta P_{l}\right.$ and $\left.\Delta P_{r}\right)$ and difference in maximum pressures $\left(\Delta_{\max } P_{l}\right.$ and $\left.\Delta_{\max } P_{r}\right)$ for different reducer diameters. Variations with respect to the $19 \mathrm{~mm}$ diameter case are computed. Length is $27 \mathrm{~mm}$.

Detailed results for the axial forces on the device ( $y$-component) and flow rates, for the different designs, are reported in Figure 12, while Table 3 summarizes the maximum and mean values, the mean flows and the pressure drops.

As the inlet imposed pressure does not change, reducing the diameter results in higher force magnitudes during systole, with a peak systole axial force varying from $5 \mathrm{~N}$, for the reducer diameter equal to $25 \mathrm{~mm}$, to $70 \mathrm{~N}$, for a diameter of $14 \mathrm{~mm}$ (see Figure 12). However, when the valve is closed, the forces in the different configurations remain very similar, with a slight decrease for the smaller diameters, due to the fact that the pressure on the side of the device facing the PAs is lower.

Due to the higher hydrodynamic resistance of the reducers with smaller diameter, the mean flows strongly decrease (up to $23 \%$ for a diameter of $14 \mathrm{~mm}$ ). Similarly, difference in maximum pressures for smaller diameters increase up to $14-15 \mathrm{mmHg}(15-16 \mathrm{mmHg}$ for the pressure drops), i.e. $+23-37 \%(15-21 \%)$ for the device with diameter $14 \mathrm{~mm}$. On the contrary, increasing the diameter has a weaker effect on the hemodynamics (Table 3): the increase of flow rate for the largest diameter is only $7 \%$ while the highest maximum pressure difference is decreased by $8 \%$ only. 


\section{Discussion}

\subsection{Effect of devices}

The device has not been implanted yet in patients. Such study thus provides insights on this novel device. The computational results show that with and without the PPVR hemodynamics in the PAs are very different. In particular, without the device the flow rate exhibits a regurgitation dynamics, typical of valve-free or valve-deficient cases [21, 32, 16, 37]. The modeled regurgitation fraction is coherent with the three available patient data, but on the higher end of reported cases $[16,37]$. The valve successfully restores the pulmonary output, lowers systolic flow from $500 \mathrm{~mL} / \mathrm{s}$ to $330 \mathrm{~mL} / \mathrm{s}$, and prevents regurgitation during diastole. The fact that the resulting inflow has these features of normal pulmonary flow validates to some extend the model. Interestingly, the device also yields a velocity field which is more regular in the MPA and across the device, reducing vorticity. Besides, the dynamics with device is more symmetric between the two PAs, with a difference in peak systolic pressure of $2-3 \mathrm{mmHg}$ only (with respect to the $5 \mathrm{mmHg}$ difference without the device). Since the outlet Windkessel parameters are the same for both PAs, this symmetrization is probably due to the difference in the three-dimensional flow structure induced by the device in the entire geometry. With the PPVR, the low velocity region at the bifurcation is more prominent due to the reducer, but vorticity is rather reduced. However, the valve eliminates the flow detachment in the posterior part of the MPA. These differences between without and with the device are in line with the 4D-MRI flow measurements of ToF patients vs. healthy controls in [16]. ToF pulmonary trunk peak velocity was twice the normal value. ToF flows were characterized by the presence of regurgitation and significant vortices. ToF flow splits denoted high asymmetry, with high variability, whereas controls had a very even flow split. It is interesting that the modeled renormalization is coherent with this patients comparison, despite not taking into account longer term adaptation that is included in the patient comparison. Modeling is thus in this regard a tool to better understand causes of these patients differences.

The valve is necessary to restore normal hemodynamics in the PA. Hence, one of the main biomedical questions motivating this study is the potential pressure gradient introduced by the device for the different designs since it reduces the conduit size. Indeed a high pressure gradient would increase right ventricular load or lower pulmonary flow, and thus mitigate the positive effect of the device. In the clinics as in the animal experiments, the pressure gradient between the RV and the PA is actually measured as systolic (maximum) pressure in the RV minus systolic (maximum) pressure in the PA (close to the bifurcation). These maxima may not occur at the same time, and thus this is with this measure that the computed results are now compared to the experimental values. The results confirm that the device does not introduce clinically significant pressure gradients, even for the tightest design. In particular, without device, the maximum pressure differences along the PA are of the order of $8-12 \mathrm{mmHg}$, increasing to $10-13 \mathrm{mmHg}$ for a full device of length 22 $\mathrm{mm}$ and diameter $19 \mathrm{~mm}$. These results agree with pressure differences experimentally observed in animals of $9 \mathrm{mmHg}( \pm 3 \mathrm{mmHg})[1], 10 \mathrm{mmHg}$ (acutely 4-16 $\mathrm{mmHg}$, after remodeling 6-24 $\mathrm{mmHg}$ ) [24], $11 \mathrm{mmHg}(8-16 \mathrm{mmHg})$ [8], considered clinically acceptable. Future study could investigate to which extend this is still the case in other patient-specific cases, for example on selected cases where a significant pressure gradient without any device is clinically seen. It could also include other physiological states, such as exercise conditions [19, 31, 38]. 
A point of biomedical interest is that introduction of the reducer alone increases pressure loss more than with the full device (PPVR). This is due to the fact that overall the reducer adds a resistance in the system, whereas the full device significantly lowers forward flows while increasing mean flow. A matter of debate in clinical research is to either introduce the full device at once, or to perform a two-step procedure first implanting the reducer, check for the pressure gradient and wait for endothelialization, and then at a later time introducing the valved-stent reducing the risk of device embolization. These results indicate that the two-step procedure may over predict the final pressure loss and introduce between the two steps a period of even worse hemodynamics conditions where pressure loss is increased and regurgitation is still present.

The reducer stability is important as it is where the valved-stent is anchored. In the latest version [24], the reducer is inserted in its final configuration into the delivery system. Through the jugular vein, and over a stiff guide wire, the delivery system is introduced and advanced into the main pulmonary artery. The device is then deployed in the target zone by unsheathing the device. Migration can then be an issue but less probable over time by fibrous tissue fixing the device on the wall. In order to investigate the short term stability of the device, the forces acting on the reducer were computed in the different configurations. For this assessment only pressure forces have been considered, which are expected to be the largest contribution to hemodynamics forces: a pressure of $10 \mathrm{mmHg}$ corresponds to almost 700 times more than the average wall-shear stress value in normal PAs (2 Pa in [31]). The maximum force, for a device of length $22 \mathrm{~mm}$ and diameter $19 \mathrm{~mm}$ is $144 \mathrm{~N}$, and occurs for all designs when the valve is closed and RV pressure is minimum, pushing the device towards the RV. In fact, during diastole the net forces on the closed valve and on the reducer are of the same order of magnitude (see Figure 9). During systole (valve open), the net force tends to shift the device up to the PA. Contrarily to first intuition, the device is pushed overall towards the RV.

\subsection{Design study}

A design study was performed, investigating the effect of geometrical parameters. Length reducer variation within the range of the experimentally tested cases [1] was considered. The results show that reducer length significantly affects neither the pressure drops nor the forces of the fluid on the device. However, a longer device would increase the friction and thus increase its stability. Finally, for the considered case, there seems to be an optimum length to achieve a minimum force on the device.

Regarding the reducer diameter, devices of diameters between $14 \mathrm{~mm}$ and $25 \mathrm{~mm}$ were considered, which corresponds to the range of devices investigated in experiments (reduction of diameter to $20 \mathrm{~mm}$ in [1], reducer of $12 \mathrm{~mm}$ [24], increased to $21.5 \mathrm{~mm}$ after valve implantation, reduction to $18 \mathrm{~mm}$ in [8]). A smaller diameter yields an increase in pressure drops as well as a decrease in mean flows. This is likely due to the increase in viscous losses as the geometry becomes more and more restrictive. At the same time, pressure forces increase in magnitude during systole (when the valve is open), while remain mainly of the same order when the valve closed. In fact there is a balance between several biomechanics components: for a given pressure, the force on the smaller diameter device is higher because of its increased area on which pressure is acting (recall that the external diameter is the same, dictated by the native geometry). But a smaller diameter also induces lower flow and lower pressure in the Windkessel, decreasing the pressure force. 
This balance explains how smaller diameters have increased maximum force during systole and on average, but decreased maximum force when the valve is closed.

\subsection{Modeling choices}

It would be interesting to compare these forces with the wall friction forces for the different surface anchorages of devices $[1,8]$. This however is beyond the scope of this paper, as this would require the detailed solid mechanics parameters of the pulmonary wall [9], stent, and complex solid-solid-fluid numerical simulations between the arterial wall, the device and blood flow (see, e.g., [3]). Thus, the reducer was considered as an impermeable fixed wall and the interaction with blood flow and arterial wall - also considered fixed- neglected, to estimate as a first approximation the order of magnitude of the hemodynamic forces.

In all the simulations, the same ventricular pressure has been imposed at the inlet. This choice is motivated by the fact that, in order to compare the hemodynamics with and without PPRV, the flow cannot be imposed as a boundary condition, as it is strongly conditioned by the presence of the valve. To avoid imposing flow or pressure, future work could involve coupling the 3D model at the inlet to a right heart lumped (OD) model. However this model should be tailored to ToF patients. Similarly, the outlet Windkessel model parameters have been kept unchanged in all configuration. The assumption is thus that there is no adaptation of the downstream vasculature due to the device implantation. Acutely this is probably reasonable, but if data would be available on such adaptation, they could be readily included in OD modeling. However since computed inlet flow rates with and without the device are coherent with valve-deficient patients versus healthy controls (mean flow rate restored to $5 \mathrm{~L} / \mathrm{min}$ ), it is expected that adding a ventricular model would not significantly change the inlet pressure. In the same line of argument, adding adaptation into the downstream model would then mainly change the magnitude of the pressure but not the pressure gradient.

As a conclusion of this study, for design purposes it does not seem necessary to have access to a full 3-dimensional velocity profile at the inlet of the 3D model, in order to assess the radial forces acting on the device or the time-variation of the forces. However, if a precise study is needed for the force, especially on a particular reducer geometry for a given patient, then a coupling with a right ventricle 3D CFD or fluid-solid interaction model might be necessary [32]. Note that from a modeling point of view, this coupling could provide another way to impose patient-specific boundary conditions at the inlet without directly imposing the velocity profile over time, which changes with the device and is therefore part of the sought solution.

\section{Conclusion}

A computational study of a novel percutaneous pulmonary valve reducer for enlarged RVOT was carried out, comparing the hemodynamics without the device, with the reducer alone or with the full device. In particular, in a typical patient setting, devices of different lengths and diameters were investigated, quantifying their effects on hemodynamics and the forces exerted on them.

The first result of this study is that the PPVR does not create a significant pressure drop according to clinical expertise, even for the narrowest considered diameters, which is in line with the measured values in animal experiments. A two-step procedure (introducing the reducer first to 
check the pressure gradient, and then after some time the valved-stent) rather than introducing the full device at once, may over predict the final pressure loss and create a period during the two steps of even worse hemodynamics conditions. Moreover, the presence of the valve efficiently palliates pulmonary regurgitation, increases pulmonary flow and regularizes the flow stream. Forces on the valve and on the reducer are of the same magnitude when the valve is closed. On average the device is pushed upstream. Hence, sliding would thus be expected to be towards the right ventricle. Varying the reducer geometrical parameters, only a slight influence of the design is observed on the mean and maximum forces within the length and diameter ranges of the existing devices, thus facilitating future device design considerations. As a general outcome, increasing the device length decreases the mean force, without changing significantly the flow rate, while the optimum case is found for an intermediate length. Decreasing the device diameter increases the mean force, and changes the maximum force in a non-monotonic way (all within $6 \%$ only from the reference configuration, which corresponded to the intermediate diameter). However the flow rate might decrease much more significantly (up to $23 \%$ for a reducer diameter of $14 \mathrm{~mm}$ ). This could potentially have a clinical impact, especially considering reducer deformation and PA remodeling after implantation.

Such study is a tool to assess functional changes to better design this new device, but in the same way minimally invasive heart valve functional replacement for pulmonary, as well as aortic or mitral valves [28]. Future work to assess in which conditions such a device would slide could include fluid-solid interaction between blood and the complex surrounding solid, and solid-solid (mechanical but also potentially biological) interaction between the reducer and the arterial wall.

\section{Acknowledgments}

The authors gratefully acknowledge Kristin McLeod, formerly at Asclepios project team, INRIA Sophia-Antipolis Mediterranée, France, now at Simula Research Laboratory, Oslo, for the device-free geometry reconstruction from MRI and Jean-Frédéric Gerbeau, REO project team, INRIA Paris-Rocquencourt, France, for initiating the SIRAP project.

A. Caiazzo, R. Guibert, Y. Boudjemline and I. E. Vignon-Clementel declare that they have no conflict of interest. No human studies were carried out by the authors for this article. No animal studies were carried out by the authors for this article.

[1] Amahzoune, B., Szymansky, C., Fabiani, J.N., Zegdi, R.: A new endovascular size reducer for large pulmonary outflow tract. European Journal of Cardio-Thoracic Surgery 37(3), 730-732 (2010)

[2] Arbia, G., Corsini, C., Baker, C., Pennati, G., Hsia, T.Y., Vignon-Clementel, I.E., for MOCHA: Pulmonary hemodynamics simulations before stage 2 single ventricle surgery: patient-specific parameter identification and clinical data assessment. Cardiovascular Engineering and Technology (2015). DOI 10.1007/s13239-015-0212-3

[3] Astorino, M., Gerbeau, J.F., Pantz, O., Traoré, K.F.: Fluid-structure interaction and multi-body contact. application to the aortic valves. Comput. Methods Appl. Mech. Eng 198(46-46), 3603-3612 (2009)

[4] Astorino, M., Hamers, J., Shadden, S., Gerbeau, J.F.: A robust and efficient valve model based on resistive immersed surfaces. Int. J. Numer. Method Biomed. Eng. 28(9), 937-959 (2012)

[5] Bertoglio, C., Caiazzo, A.: A tangential regularization method for backflow stabilization with application to blood flow simulations. Journal Comp. Phys. 261(1800), 162-171 (2013)

[6] Bonhoeffer, P., Boudjemline, Y., Saliba, Z., Hausse, A., Aggoun, Y., Bonnet, D., Sidi, D., Kachaner, J.: Transcatheter implantation of a bovine valve in pulmonary position: a lamb study. Circulation 102(7), 813816 (2000) 
[7] Botney, M.D.: Role of hemodynamics in pulmonary vascular remodeling: implications for primary pulmonary hypertension. Am J Respir Crit Care Med 159(2), 361-4 (1999). DOI 10.1164/ajrccm.159.2.9805075

[8] Boudjemline, Y., Agnoletti, G., Bonnet, D., Sidi, D., Bonhoeffer, P.: Percutaneous pulmonary valve replacement in a large right ventricular outflow tract: an experimental study. J Am Coll Cardiol 43(6), $1082-7$ (2004). DOI 10.1016/j.jacc.2003.10.037

[9] Cabrera, M., Oomens, C., Bouten, C., Bogers, A., Hoerstrup, S., Baaijens, F.: Mechanical analysis of ovine and pediatric pulmonary artery for heart valve stent design. Journal of biomechanics 46(12), 2075-2081 (2013)

[10] Caiazzo, A., Fernández, M., Gerbeau, J.F., Martin, V.: Projection schemes for fluid flows through a porous interface. SIAM J. Sci. Comp. 33(2), 541-564 (2011)

[11] Chorin, A.: Numerical solution of the Navier-Stokes equations. Math. Comp. 22, 745-762 (1968)

[12] Das, A., Gottliebson, W.M., Karve, M., Banerjee, R.: Comparison of hemodynamic endpoints between normal subject and tetralogy patient using Womersley velocity profile and MR based flow measurements. Mol. \& Cell. Biomech. 8(1), 21-42 (2011)

[13] Fernández, M., Gerbeau, J.F., Martin, V.: Numerical simulation of blood flows through a porous interface. Math. Mod. Num. An. (M2AN) 42(6), 961-990 (2008)

[14] Fogel, M.A., Sundareswaran, K.S., de Zelicourt, D., Dasi, L.P., Pawlowski, T., Rome, J., Yoganathan, A.P.: Power loss and right ventricular efficiency in patients after tetralogy of fallot repair with pulmonary insufficiency: Clinical implications. Journal of Thoracic and Cardiovascular Surgery 143(6), 1279-1285 (2012). DOI 10.1016/j.jtcvs.2011.10.066

[15] Frank, O.: Die Grundform Des Arteriellen Pulses. Zeitung für Biologie 37, 483-586 (1899)

[16] Geiger, J., Markl, M., Jung, B., Grohmann, J., Stiller, B., Langer, M., Arnold, R.: 4d-mr flow analysis in patients after repair for tetralogy of fallot. European Radiology 21, 16511657 (2011)

[17] George, P., Borouchaki, H., Saltel, E.: Ultimate robustness in meshing an arbitrary polyhedron. international journal for numerical methods in engineering. IJNME 58, 1061-1089 (2003)

[18] Guermond, J.L., Minev, P., Shen, J.: An Overview of Projection methods for incompressible flows. Comput. Methods Appl. Mech. Engrg. 195, 6011-6045 (2006)

[19] Guibert, R., Mcleod, K., Caiazzo, A., Mansi, T., Fernández, M.A., Sermesant, M., Pennec, X., VignonClementel, I.E., Boudjemline, Y., Gerbeau, J.F.: Group-wise construction of reduced models for understanding and characterization of pulmonary blood flows from medical images. Medical Image Analysis 18(1), 63-82 (2014)

[20] H. Si: TetGen: A Quality Tetrahedral Mesh Generator and a 3D Delaunay Triangulator. Tech. Rep. 1762, WIAS, Berlin (2013)

[21] Kilner, P.J., Balossino, R., Dubini, G., Babu-Narayan, S.V., Taylor, A.M., Pennati, G., Migliavacca, F.: Pulmonary regurgitation: The effects of varying pulmonary artery compliance, and of increased resistance proximal or distal to the compliance. Int. J. Cardiology 133(2), 157-166 (2009). DOI 10.1016/j.ijcard.2008.06.078

[22] Lurz, P., Bonhoeffer, P., Taylor, A.M.: Percutaneous pulmonary valve implantation: an update. Expert Rev Cardiovasc Ther 7(7), 823-33 (2009). DOI 10.1586/erc.09.57

[23] McLeod, K., Caiazzo, A., Fernández, M.A., Mansi, T., Vignon-Clementel, I.E., Sermesant, M., Pennec, X., Boudjemline, Y., Gerbeau, J.F.: Atlas-Based Reduced Models of Blood Flows for Fast Patient-Specific Simulations. In: Proc. MICCAI Workshop on Statistical Atlases and Computational Models of the Heart: Mapping Structure and Function + a Cardiac Electrophysiological Simulation Challenge (STACOM+CESC'10), LNCS, vol. 6364, pp. 95-104. Springer, Beijing (2010)

[24] Mollet, A., Basquin, A., Stos, B., Boudjemline, Y.: Off-pump replacement of the pulmonary valve in large right ventricular outflow tracts: A transcatheter approach using an intravascular infundibulum reducer. Pediatric Research 62(4), 428-433 (2007)

[25] Momenah, T.S., El Oakley, R., Al Najashi, K., Khoshhal, S., Al Qethamy, H., Bonhoeffer, P.: Extended application of percutaneous pulmonary valve implantation. J Am Coll Cardiol 53(20), 1859-63 (2009). DOI 10.1016/j.jacc.2008.08.061

[26] Pant, S., Fabrèges, B., Gerbeau, J.F., Vignon-Clementel, I.: A methodological paradigm for patient-specific multi-scale cfd simulations: from clinical measurements to parameter estimates for individual analysis. International journal for numerical methods in biomedical engineering 30(12), 1614-1648 (2014) 
[27] Prasad, A., To, L.K., Gorrepati, M.L., Zarins, C.K., Figueroa, C.A.: Computational analysis of stresses acting on intermodular junctions in thoracic aortic endografts. J. Endovasc. Ther. 18(4), 559-68 (2011). DOI 10.1583/113472.1

[28] Preston-Maher, G.L., Torii, R., Burriesci, G.: A technical review of minimally invasive mitral valve replacements. Cardiovascular Engineering and Technology (2014)

[29] Schievano, S., Coats, L., Migliavacca, F., Norman, W., Frigiola, A., Deanfield, J., Bonhoeffer, P., Taylor, A.M.: Variations in right ventricular outflow tract morphology following repair of congenital heart disease: implications for percutaneous pulmonary valve implantation. J Cardiovasc Magn Reson 9(4), 687-95 (2007). DOI $10.1080 / 10976640601187596$

[30] Schievano, S., Taylor, A.M., Capelli, C., Lurz, P., Nordmeyer, J., Migliavacca, F., Bonhoeffer, P.: Patient specific finite element analysis results in more accurate prediction of stent fractures: application to percutaneous pulmonary valve implantation. J Biomech 43(4), 687-93 (2010). DOI 10.1016/j.jbiomech.2009.10.024

[31] Tang, B.T., Fonte, T.A., Chan, F.P., Tsao, P.S., Feinstein, J.A., Taylor, C.A.: Three-dimensional Hemodynamics in the Human Pulmonary Arteries under Resting and Exercise Conditions. Annals of Biomedi 39(1), 347-58 (2011)

[32] Tang, D., Yang, C., Geva, T., Del Nido, P.J.: Patient-specific mri-based 3d fsi rv/lv/patch models for pulmonary valve replacement surgery and patch optimization. J Biomech Eng 130(4), 041,010 (2008). 18601452

[33] Temam, R.: Une méthode d'approximation de la solution des équations de Navier-Stokes. Bull. Soc. Math. France 96, 115-152 (1968)

[34] Troianowski, G., Taylor, C.A., Feinstein, J.A., Vignon-Clementel, I.E.: Three-Dimensional Simulations in Glenn Patients: Clinically Based Boundary Conditions, Hemodynamic Results and Sensitivity to Input Data. J. Biomech. Engnr.-Trans. Asme 133(11) (2011). DOI 111006 10.1115/1.4005377

[35] Vignon-Clementel, I., Figueroa, C., Jansen, K., Taylor, C.: Outflow boundary conditions for 3D simulations of non-periodic blood flow and pressure fields in deformable arteries. Comp. Meth. Biomech. Biomed. Engnr. 111(3), 502-513 (2010)

[36] Vignon-Clementel, I.E., Marsden, A.L., Feinstein, J.A.: A primer on computational simulation in congenital heart disease for the clinician. Progr. Ped. Card. 30(1-2), 3-13 (2010). DOI 10.1016/j.ppedcard.2010.09.002

[37] Voser, E.M., Kellenberger, C.J., Buechel, E.R.V.: Effects of Pulmonary Regurgitation on Distensibility and Flow of the Branch Pulmonary Arteries in Tetralogy of Fallot. Pediatric Cardiology 34, 1118-1124 (2013)

[38] Yang, W., Vignon-Clementel, I.E., Troianowski, G., Reddy, V.M., Feinstein, J.A., Marsden, A.L.: Hepatic blood flow distribution and performance in conventional and novel Y-graft Fontan geometries: A case series computational fluid dynamics study. J. Thorac. Cardiovasc. Surg. (2011). DOI S0022-5223(11)00944-5 [pii] 10.1016/j.jtcvs.2011.06.042

[39] Yun, B.M., Dasi, L.P., Aidun, C.K., Yoganathan, A.P.: Computational modelling of flow through prosthetic heart valves using the entropic lattice-boltzmann method. Journal of Fluid Mechanics 743, 170-201 (2014). DOI 10.1017/jfm.2014.54 